\title{
Terapias utilizadas em gestantes que apresentam Transtorno Depressivo Maior e suas implicações no pós-parto
}

\author{
Therapies used in pregnant women who present Major Depressive Disorder and its postpartum \\ implications
}
Terapias utilizadas en mujeres embarazadas que presentan Trastorno Depresivo Mayor y sus implicaciones postparto

\author{
Rafael d'Oliveira Batista Silva \\ ORCID: https://orcid.org/0000-0001-5352-4586 \\ Centro Universitário Christus, Brasil \\ E-mail: rafa_silva_1995@hotmail.com \\ Mardenia Gomes Vasconcelos Pitombeira \\ ORCID: https://orcid.org/0000-0003-2969-6526 \\ Centro Universitário Christus, Brasil \\ E-mail: mardenia.pitombeira@unichristus.edu.br \\ Iris Cristina Maia Oliveira \\ ORCID: https://orcid.org/0000-0001-9958-7320 \\ Faculdade Rodolfo Teófilo, Brasil \\ E-mail: iriscmo@gmail.com \\ Aline de Souza Pereira \\ ORCID: https://orcid.org/0000-0001-9316-5987 \\ Centro Universitário Christus, Brasil \\ E-mail: szp.aline@gmail.com \\ Francisca Taciana Sousa Rodrigues Maia \\ ORCID: https://orcid.org/0000-0001-6764-9363 \\ Centro Universitário Christus, Brasil \\ Instituto Dr. José Frota, Brasil \\ E-mail: taciana7maia@gmail.com
}

\begin{abstract}
Resumo
Objetivo: Debater sobre as terapias mais utilizadas em gestantes que apresentam TDM e analisar as implicações pósparto, além dos riscos e benefícios das drogas de escolha. Metodologia: Revisão integrativa da literatura, utilizando artigos das plataformas acadêmicas SciELO, PuBMED, MEDLINE e Science Direct, considerando um intervalo entre 2016-2021, que abordassem o assunto da terapia farmacológica com Inibidores Seletivos de Recaptação de Serotonina (ISRS) e/ou Inibidores Seletivos de Recaptação de Serotonina e Noradrenalina (ISRSN) no período gestacional ou ainda a não adesão ao tratamento, tendo um achado de 12 artigos que atenderam aos critérios de inclusão e exclusão. Resultados: Os estudos, em sua maioria, corroboram com a eficácia do tratamento com ISRS e /ou ISRSN em gestantes, porém existe dificuldade em elaborar um plano com o menor risco possível, isso porque os medicamentos com melhor resultado também apresentam certo grau de risco. Esses mesmos estudos, assim como outros, destacam os efeitos teratogênicos e alguns outros achados durante tratamentos com ISRS e/ou ISRSN e, em caso do não tratamento, os efeitos da depressão ao longo da gestação. Alguns dos achados indicam a adesão ao cotratamento utilizando-se de terapias não farmacológicas, como a psicoterapia (recomendada por guidelines como o tratamento inicial ideal em casos leves a moderados), associadas com a terapia farmacológica. Conclusões: Pode-se concluir que existem estudos sobre os benefícios e malefícios do uso de antidepressivos durante a gestação, de tal forma que dificulta definir a melhor estratégia, cabendo aos profissionais definir custo-benefício de acordo com cada paciente e situação.
\end{abstract}

Palavras-chave: Transtorno depressivo maior; Depressão pós-parto; Responsividade materna; Inibidores seletivos de recaptação de serotonina; Enfermagem.

\footnotetext{
Abstract

Objective: Debate on the therapies most used in pregnant women with MDD and analyze the postpartum implications, in addition to the risks and benefits of the drugs of choice. Methodology: Integrative literature review, using articles from the academic platforms SciELO, PuBMED, MEDLINE and Science Direct, considering an interval between 2016-2021, that addressed the subject of pharmacological therapy with Selective Serotonin Reuptake Inhibitors
} 
(SSRIs) and/or Serotonin Norepinephrine Reuptake Inhibitors (SNRIs) in the gestational period or non-adherence to treatment, with a finding of 12 articles that met the inclusion and exclusion criteria. Results: Most studies support the effectiveness of treatment with SSRIs and/or SNRIs in pregnant women, but there is difficulty in developing a plan with the lowest possible risk, because the drugs with the best results also present a certain degree of risk. These same studies, as well as others, highlight the teratogenic effects and some other findings during treatments with SSRIs and/or SNRIs and, in case of non-treatment, the effects of depression throughout pregnancy. Some of the findings indicate adherence to co-treatment using non-pharmacological therapies, such as psychotherapy (recommended by guidelines as the ideal initial treatment in mild to moderate cases), associated with pharmacological therapy. Conclusions: It can be concluded that there are studies on the benefits and harms of the use of antidepressants during pregnancy, in such a way that it is difficult to define the best strategy, it being up to professionals to define costbenefit according to each patient and situation.

Keywords: Major depressive disorder; Postpartum depression; Maternal responsiveness; Selective serotonin reuptake inhibitors; Nursing.

\section{Resumen}

Objetivo: Debatir las terapias más utilizadas en gestantes con TDM y analizar las implicaciones posparto, además de los riesgos y beneficios de los fármacos de elección. Metodología: Revisión integrativa de la literatura, utilizando artículos de las plataformas académicas SciELO, PuBMED, MEDLINE y Science Direct, considerando un intervalo entre 2016-2021, que abordaron el tema de la terapia farmacológica con Inhibidores Selectivos de la Recaptación de Serotonina (ISRS) y / o Inhibidores de Serotonina. y Recaptación de Noradrenalina (IRSN) durante el embarazo o no adherencia al tratamiento, con un hallazgo de 12 artículos que cumplieron con los criterios de inclusión y exclusión. Resultados: La mayoría de estudios corroboran la efectividad del tratamiento con ISRS y / o IRSN en mujeres embarazadas, pero existe dificultad para desarrollar un plan con el menor riesgo posible, porque los fármacos con mejores resultados también presentan cierto grado de riesgo. Estos mismos estudios, entre otros, destacan los efectos teratogénicos y algunos otros hallazgos durante los tratamientos con ISRS y / o IRSN y, en caso de no tratamiento, los efectos de la depresión durante el embarazo. Algunos de los hallazgos indican adherencia al cotratamiento mediante terapias no farmacológicas, como la psicoterapia (recomendada por las guías como tratamiento inicial ideal en casos leves a moderados), asociada a la terapia farmacológica. Consideraciones finales: Se puede concluir que existen estudios sobre los beneficios y perjuicios del uso de antidepresivos durante el embarazo, de tal manera que es difícil definir la mejor estrategia, quedando a los profesionales definir el costo-beneficio según cada paciente y situación.

Palabras clave: Trastorno depresivo mayor; Depresión post-parto; Capacidad de respuesta materna; Inhibidores selectivos de la recaptación de serotonina; Enfermería.

\section{Introdução}

Segundo a Organização Mundial da Saúde (OMS, 2020), o Transtorno Depressivo Maior (TDM) mantém-se como o principal fator de incapacidade laboral em todo o mundo, principalmente em regiões como Canadá e Estado Unidos (Albert, 2015), e responsável pela incidência de suicídios que ocupa, atualmente, a segunda posição em casos de óbitos em pessoas entre 15 e 29 anos de idade.

A taxa de prevalência continua a mesma, sendo o sexo feminino mais afetado que o sexo masculino, a hipótese para essa ocorrência é baseada nas flutuações hormonais que ocorrem durante a vida de uma mulher, principalmente nas mulheres jovens onde as alterações são mais bruscas devido o ciclo menstrual, sendo a puberdade considerada um divisor e por isso mulheres jovens possuírem maior incidência (Albert, 2015). Porém, Gordon e Girdler (2014) levantam a hipótese dos hormônios ovarianos, principalmente o estrógeno, atuarem como um protetor do processo depressivo apontando, ainda, o período de mudança entre perimenopausa e pós-menopausa como um momento crítico para surgimento do transtorno depressivo (caracterizado como depressão pós-menopausa) devido à diminuição da captação de serotonina em seus receptores e eventual redução do volume do hipocampo.

Os sintomas principais dos transtornos depressivos são o humor triste ou deprimido e/ou anedonia, além desses o indivíduo pode apresentar perda ou ganho considerável de peso, insônia, agitação ou retardo, diminuição na concentração, sentimentos de inutilidade ou culpa excessiva e, ainda, intenções suicidas (APA, 2014).

O ponto a ser debatido durante esse estudo é acerca das terapias mais utilizadas em gestantes que apresentam TDM e analisar as implicações pós-parto, além dos riscos e benefícios das drogas de escolha na terapia farmacológica. Atualmente, as 
principais escolhas para o tratamento são os Inibidores Seletivos de Recaptação de Serotonina (ISRS), sendo a sertralina o primeiro pscotrópico da lista (Carvalho, et al., 2020).

A gestação é um momento da vida muito aguardado por algumas mulheres e, como outros momentos, gera picos de emoções. A mulher costuma apresentar ansiedade e medo, sente-se insegura e ainda é obrigada a ouvir diversos comentários e conselhos a respeito desse período, de como proceder e o que pode ou não, além da reorganização de vida e costumes para essa futura mãe (Klieman, Böing, \& Crepaldi, 2017; Krob, et al., 2017).

Esse contexto citado acima somado com as flutuações hormonais e alterações no corpo materno incidem em um aumento no risco de gestantes apresentarem depressão (Klieman, Böing, \& Crepaldi, 2017; Krob, et al., 2017; Silva, et al, 2020), contrapondo a crença popular da gestação ser um período de felicidade, de realização e do alcance de um sonho, além de aumentar a chance da mulher se manter depressiva no período pós-parto (Silva, et al., 2020).

O transtorno depressivo no pós-parto é, geralmente, decorrente de depressão já no pré-natal, e decorre habitualmente por um período de 12 meses após o parto. As consequências para essa puérpera, devido à enorme carga de ansiedade e estresses psicológicos vivenciados durante a gestação, serão o risco e desenvolver outros transtornos psquiátricos, menor autoestima e menos confiança em seu papel parenteral, acarretando em problemas de saúde física, propensão a atos autolesivos e diminuição no autocuidado (Fonseca, \& Canavarro, 2017). Já para o recém-nascido (RN), segundo Klieman, Böing \& Crepaldi (2017), pode resultar em parto pré-termo, RN de baixo peso e retardos no desenvolvimento neurológico do mesmo, sendo visualizado na regulação da atenção, do temperamento e do emocional e no desenvolvimento motor e cognitivo.

O tratamento para o TDM em puérperas tem sido realizado através de Terapia Cognitivo-Comportamental e de Terapia Interpessoal devido à eficácia. Porém, a terapia medicamentosa é, também, muito importante e realizada com os ISRS sendo a sertralina, a paroxetina e a fluvoxamina as drogas de primeira escolha devida a eficácia comprovada e baixa toxicidade (Fonseca, \& Canavarro, 2017).

Devido o aumento da incidência de casos de depressão na atualidade e do sexo feminino ser um fator de risco para esse adoecimento, faz-se necessário uma atenção redobrada no atendimento de gestantes, principalmente na estratégia do prénatal, tendo como foco identificar casos de transtornos depressivos ou de potenciais casos e acompanhar, de forma integral e resolutiva, essa mulher. Para tal, os profissionais devem saber realizar o acolhimento desse público e compreender "quais os riscos e benefícios da terapia medicamentosa para gestantes e puérperas?”

Em relação ao tratamento medicamentoso, acredita-se que os ISRS permanecem como as drogas de primeira escolha, porém é preciso determinar quais outras drogas possuem equivalente ou parecida eficácia a essa categoria para aquelas pacientes que possam apresentar reações de hipersensibilidade com algum dos componentes dos ISRS. Já em relação à detecção precoce, é levantada a hipótese de que o diagnóstico precoce durante consultas de pré-natal pode diminuir consideravelmente quadros de depressão pós-parto, diminuindo os efeitos negativos tanto na puérpera quanto no RN.

Devido o exposto acima, o presente trabalho tem como intuito debater sobre as terapias mais utilizadas em gestantes que apresentam TDM e analisar as implicações pós-parto, além dos riscos e benefícios das drogas de escolha.

\section{Metodologia}

Trata-se de uma revisão integrativa da literatura, que tem como propósito reunir informações de diferentes textos bibliográficos, de forma a aproximar os conhecimentos acerca dos fatores que comprovam a relação tratada nessa pesquisa (Mendes, Silveira, \& Galvão, 2008). A busca de artigos, tanto na língua portuguesa como na língua inglesa, foi realizada nas bases de dados da Biblioteca Virtual de Saúde (BVS) e Google Acadêmico, priorizando as plataformas acadêmicas como SciELO, PubMed., MEDLINE, Biblioteca Digital de Periódicos e Science Direct. O período de coleta compreendeu os anos de Julho de 2020 a maio de 2021. 
Os critérios de inclusão foram textos completos, disponíveis online, podendo estar na língua portuguesa ou inglesa e que abordassem o uso de ISRS, ISRSN e a não utilização de terapia medicamentosa em gestantes com TDM. Os critérios de exclusão foram textos resumidos ou incompletos, cartas, editoriais, artigos de opinião, comentários e textos bibliográficos que não abordassem o uso de ISRS, ISRSN e/ou a não terapia em gestantes com TDM. Na primeira busca foram utilizados os descritores depressão; gravidez; e antidepressivo, sem aplicar filtros obtive 856 resultados na BVS e 5.880 no Google Acadêmico. Ao aplicar o filtro de texto completo e o intervalo de ano entre 2016 e 2021, passei a ter 210 resultados na BVS e 2.010 no Google Acadêmico. Ao aplicar os critérios de inclusão, passamos a ter 117 resultados na BVS e 36 no Google Acadêmico. Após leitura de resumos, foram selecionados 11 textos na BVS e apenas 1 no Google Acadêmico.

Figura 1 - Prisma da busca metodológica dos artigos que embasassem o estudo.

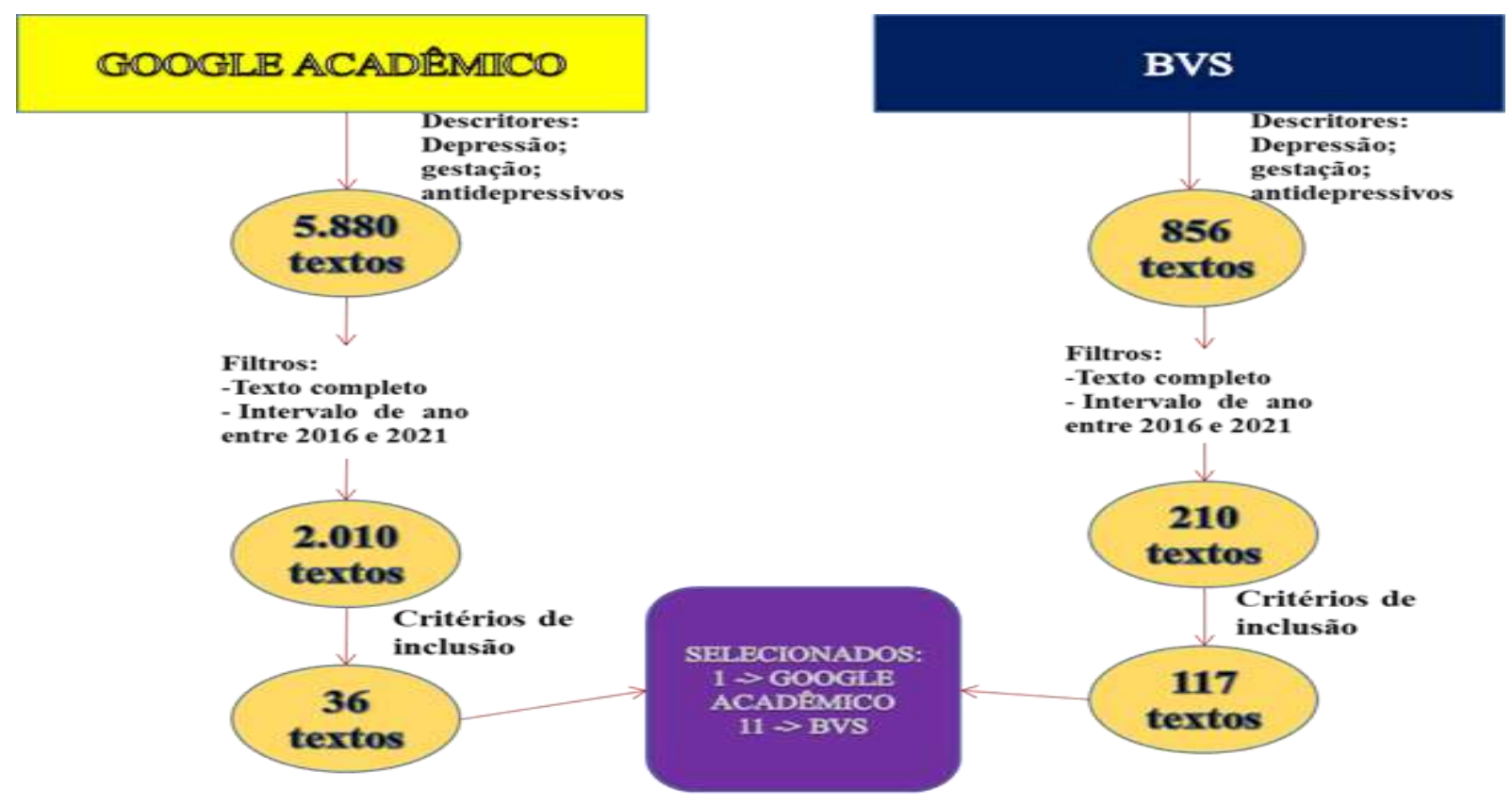

\section{Resultados}

Nossa busca resultou em 6.736 potenciais textos bibliográficos para o estudo nas bases de dados da Biblioteca Virtual de Saúde (BVS) e Google Acadêmico, priorizando PubMed, Science Direct, MEDLINE e SciELO, sendo filtrados por intervalo de ano entre 2016 e 2021 e textos que fossem completos, dispensando os textículos, tendo 2.220 resultados. Com a utilização dos critérios de inclusão e exclusão, passaram a ser 153 artigos. Foram selecionados 12 textos após avaliação dos títulos e dos resumos (Figura 1).

Os textos bibliográficos foram compilados na tabela abaixo trazendo os autores dos estudos e os resultados de cada um. Essa disposição em tabela facilita a leitura e entendimento de cada achado. Além disso, foram organizados em uma sequência lógica no qual os trabalhos se complementam em ideia (Quadro 1). 
Quadro 1 - Artigos selecionados e seus respectivos resultados.

\section{ESTUDOS}

Zhao, X., Liu, Q., Cao, S., Pang, J., Zhang, H., Feng, T. et al. (2017)

Hutchison, S. M., Masse, L. C., Pawluski, J. L., \& Oberlaner, T. F. (2018)

\section{RESULTADOS}

Estudos demonstram que o uso de antidepressivos durante período gestacional, mais especificamente os ISRS, estejam relacionados com um nível de desenvolvimento fetal considerado "abaixo do ótimo", que seria como "não é o melhor possível". O baixo peso ao nascer e o tamanho pequeno para a idade gestacional são os parâmetros usados para determinar o nível de exposição associados ao nível de "abaixo do ótimo".

Esse estudo trata da relação entre o uso de ISRS e neonatos com baixo peso ao nascer, o que fortalece os achados do estudo acima. Além disso, ele traz um dado importante referente a não relação entre mulheres com desordens psiquiátricas não medicalizadas com antidepressantes e o baixo peso ao nascer, sendo mais um indício da relação entre ISRS-peso ao nascer. Em contrapartida, relata não haver comprovação suficiente em relação ao uso de ISRS e o comprometimento do tamanho para idade gestacional.

Guan, H-B., Wei, Y., Wang, L-L., Qiao, C., \& Liu, C-X. (2018)

A pesquisa trata da possível relação entre o uso de ISRS durante o período gestacional e o aumento de risco para desenvolvimento de hipertensão gestacional e pré-eclampsia. Apesar dele não conseguir estabelecer relação entre o uso de outros antidepressivos e as duas desordens hipertensivas acima ou da continuação ou descontinuação do uso da terapia farmacológica, os dados encontrados fundamentam bem o objetivo do trabalho.

Gao, S-Y., Wu, Q-J., Sun, C., Zhang, T-N., Os dados demonstram que os ISRS apresentam aumento de risco para: Shen, Z-Q., Liu, C-X. et al. (2018) defeitos de septo miocárdico (septo atrial e ingurgitamento de trato ventricular direito); defeitos de tubo neural; doença cística no fígado e defeito de parede abdominal, onfalocele e gastrosquise; defeitos do sistema urinário; defeitos de face, olhos, orelhas, clubfoot e hipospádia; defeitos do sistema respiratório. Esses riscos foram referentes a medicalização com ISRS durante o primeiro trimestre de gestação.

Singal, D., Chateau, D., Struck, S., Lee, J. B., Dahl, M., Derksen, S. et al. (2020)

O estudo tem como intuito alavancar o impacto do uso de antidepressivos durante período gestacional através da avaliação de crianças em idade de 3 a 5 anos (jardim de infância). A avaliação considera 5 domínios: (1) boa saúde física (2) desenvolvimento cognitivo e linguagem (3) competência social e maturidade emocional (4) habilidades de comunicação (5) conhecimentos gerais. Filhos de mulheres expostas a uma prescrição de ISRS ou ISRSN apresentaram vulnerabilidade de desenvolvimento em 2 ou mais desses domínios. Exposição a pelo menos duas prescrições de ISRS ou ISRSN demonstraram vulnerabilidade dos domínios de linguagem e de desenvolvimento cognitivo.

Masarwa, R., Bar-Oz, B., Gorelik, E., Reif, S., $\quad$ O texto aborda a incidência de Hipertensão Pulmonar Persistente em Perlman, A., \& Matok, I. (2019) Neonatos (HPPN) de gestantes tratadas com ISRS ou ISRSN. Segundo os dados, o filho de uma mulher tratada durante toda a gestação com ISRS ou ISRSN apresenta um risco aumentado para HPPN. O estudo revela, ainda, que a Sertralina figura como o psicotrópico com menor risco para HPPN. Em casos de início tardio do tratamento com ISRS ou ISRSN (>20 semanas), o risco para HPPN tem incidência significativamente alta.

Vallee, J., Wong, Y., Mannino, E., Nordeng, H., \& Lupattelli, A. (2021)

Esse estudo debate acerca do uso ou do não uso de antidepressivos por parte de mulheres que estiveram grávidas. As mulheres entrevistadas que afirmaram ter feito uso de antidepressivos durante a gestação possuíam nível de escolaridade maior do que as não medicadas e possuíam ocupação (um emprego). As não medicadas apresentaram maior incidência de abuso de álcool e apresentaram sintomas depressivos mais fortes. Os ISRS foram os mais utilizados (predominância da Sertralina) seguidos da Venlafaxina dos ISRSN. Outro ponto abordado, é que mulheres medicadas apresentaram maior número de episódios de ideação de suicídio ou machucar a si mesmo (IMSM) durante o pós-parto. Em contrapartida, mulheres medicadas apresentaram maior incidência para abstenção total de IMSM no pós-parto se comparados com mulheres não medicadas. Mulheres que não fizeram o uso completo da terapia apresentaram maior incidência de ISMS no pós-parto se comparado com mulheres que fizeram uso da terapia por toda a gestação.

Berard, A., Sheehy, O., Zhao, J-P., Chambers, O estudo trata de gestantes com desordens de humor (prevalência de 
TDM) que fizeram uso continuado de antidepressivos durante gestação ou que abandonaram ou não utilizaram. Segundo os dados, o grupo que abandonou a terapia apresentou a maior prevalência de sintomas depressivos durante primeiro e segundo trimestre. $\mathrm{O}$ grupo não medicado apresentou prevalência de sintomas depressivos pelo segundo trimestre. Além disso, a modificação de dosagem durante terapia apresentou-se mais eficaz do que a não modificação.

Carvalho, L. A. G., Godoy, J. T., Baldo, A. A., Fortes, B. C. R., Lopes, D. C. S., \& Noia, D. M., 2020

Brown, H. K., 2018

Pawluski, J. L., \& Gemmel, M. (2018)

Molenaar, N. M., Kamperman, A. M., Boyce, P., \& Bergink, V. (2018)
Aponta para a classe de ISRS como as drogas de principal escolha para TDM em gestantes. Abordam, ainda, os efeitos adversos da classe e de outras classes como os ISRSN e os tricíclicos. Outro ponto discutido é o não tratamento que acarreta em danos significativos para o binômio mãefeto (alterações de fluxo arterial do feto, restrição de crescimento intrauterino, prematuridade e, até mesmo, suicídio e infanticídio) e a interrupção do tratamento, devido medo por parte da gestante, apresentou maior recidiva dos sintomas depressivos.

O trabalho reforça os achados de outros estudos quanto à restrição de crescimento fetal, prematuridade e problemas de desenvolvimento mental nas crianças, relacionados ao quadro de depressão pré-natal e alto nível de ansiedade. Por outro lado, alguns estudos demonstraram inconsistência de resultados no que diz respeito da exposição ao ISRS e problemas de desenvolvimento mental na criança, mesmo que a maioria dos estudos aponte para achados mistos com ocorrências de autismo, retardo no desenvolvimento motor, dificuldade social e problemas internos.

A autora trata ainda do tempo de exposição aos ISRN em diferentes períodos da gestação e os possíveis efeitos: exposição tardia do feto (gestação tardia, maior do que 29 semanas) demonstra aumento no risco de ansiedade ou comportamentos depressivos por volta dos 5 anos de idade.

O referente estudo também expõe os achados clínicos de efeitos adversos durante o uso de antidepressivos, principalmente ISRS, dos quais: déficit de atenção, desordens neuropsiquiátricas e desordens de desenvolvimento neural.

O mais interessante do trabalho é a relação estabelecida entre os efeitos comuns do uso de ISRS e a importância do hipocampo para essas alterações. É debatido sobre o stress já vivenciado no período gestacional devido redução fisiológica de serotonina circulante no espaço hipocampal. Quando somado ao bloqueio proferido pelos ISRS nos transportadores de serotonina, a quantidade de serotonina circulante no hipocampo é ainda mais reduzida, o que aumenta os níveis de stress.

Além disso, pelo ISRS estar presente tanto na placenta quanto no leite materno (mesmo que em menor quantidade), a mulher em período gestacional e lactante passa cargas de antidepressivos ao feto, influenciando no desenvolvimento neural.

Estudo que realiza uma busca em guidelines que tratem do uso de terapias farmacológicas ou não farmacológicas em mulheres com transtornos mentais e que pensem em engravidar, que estejam gestantes ou puérperas. Para mulheres que planejam engravidar, três guidelines desencorajam a mudança de antidepressivos, sendo aconselhada a continuação se paciente estável. Caso paciente apresente nova sintomatologia, é recomendado o início da psicoterapia (APA; COPE; NICE). Se caso mais grave, iniciar com antidepressivos (NICE; COPE). $\mathrm{Na}$ gestação, quatro guidelines recomendam a continuação do antidepressante, cinco não desaconselham a continuação, mas não especificam e três desaconselham a troca de antidepressivos. Pelo Danish, Paroxetina e Fluvoxetina são drogas a serem trocadas caso sejam os antidepressivos utilizados pela gestante. A psicoterapia é a mais indicada como terapia inicial em casos leves a moderados e antidepressivos como o tratamento inicial de escolha em casos mais graves. A ACOG é a única que aconselha antidepressivos como terapia inicial independente da gravidade do sintoma.

No pós-parto, é aconselhada a continuação da terapia e antidepressivos. Em suma, os guidelines não recomendam o uso de Paroxetina por estar associada ao aumento do risco de má-formação congênita cardiovascular em RN. Já a fluvoxetina é desaconselhada devido sua meia-vida longa e poder ser encontrada no leite materno. 


\section{Discussão}

Nas pesquisas de Field, Diego e Hernandez-Reif (2006) muitos dos artigos, que abordavam a terapia medicamentosa de adoecimentos mentais, já debatiam o uso dos psicotrópicos no pré-natal e tinham como conclusão ineficácia. Outros traziam efeitos adversos para o RN, como escore de apgar baixo e baixo desenvolvimento infantil (escala de Bayley baixa).

Estudos e exames clínicos do uso de psicotrópicos são realizados constantemente a fim de determinar risco-benefício dos tratamentos, inclusive na gestação. Apesar de alguns desses evidenciarem riscos na gravidez fazendo com que o tratamento para doenças mentais nesses casos seja clinicamente desaconselhado, especialmente no primeiro trimestre, estudos mais recentes garantem que a maioria das drogas psicotrópicas é segura para gestante e feto, sendo super indicados quando o adoecimento mental representa risco iminente para ambos, inclusive suicídio e infanticídio (Chilsom, \& Payne, 2016).

Vale ressaltar que a literatura utilizada nesse estudo possui limitações devidas múltiplos motivos, são estes: dosagens diferentes por causa da diferença corporal entre as mulheres, tais qual o peso e o metabolismo; pelo fato das condições psiquiátricas serem consideradas menos sérias à saúde do que outros adoecimentos, tornando os psicotrópicos menos necessários e considerados um custo adicional desnecessário; a impossibilidade de avaliar os efeitos da terapia em longo prazo no recém-nascido; fatores de risco como diabetes, tabagismo, obesidade e uso indevido de substâncias são comuns em pacientes com desordens de humor, mais do que na maioria da população. Estudos que não consideram a associação desses fatores de risco e do adoecimento psiquiátrico podem demonstrar efeitos adversos, no feto, não propriamente oriundos do uso dos psicotrópicos, mas da presença desses riscos para gestantes (Chilsom, \& Payne, 2016; Field, Diego, \& Hernandez-Rief, 2010).

Os ISRS são a classe mais utilizada e que demonstra melhores resultados na terapia durante a gravidez, sendo eles a fluvoxamina, paroxetina, sertralina, fluoxetina, citralopam e escitralopam (Carvalho, et al., 2020; Balkowiec-Iskra, et al., 2017). Como método alternativo, tem-se os Inibidores Seletivos de Recaptação da Serotonina e da Noradrenalina (ISRSN) (Balkowiec-Iskra, et al., 2017).

No que diz respeito aos ISRSN, a venlafaxina é a droga de escolha da classe, porém demonstrou resultados em que gestantes tratadas apresentaram risco aumentado para cianose, dispnéia, hipoglicemia, convulsões, tremores, hiperreflexia, hipotonia, irritabilidade, hipertonia e choro persistente, além de hipertensão gestacional, pré-eclampsia, aumento do intervalo QT no recém nascido e da mortalidade neonatal (Pearlstein, 2013; Carvalho, et al., 2020). Já quanto aos antidepressivos tricíclicos, tendo amitriptilina e imipramina como drogas de referência, não foram associadas malformações, porém o uso durante o primeiro trimestre pode acarretar em teratogenicidade congênita (Goracci, et al., 2015; Carvalho, et al., 2020). Existem poucos estudos abordando essas duas classes e outras mais, sendo consideradas irrelevantes a quantidade de evidências para categorizá-las como opções melhores que os ISRS.

As drogas dos ISRS foram associadas ao aumento do risco de Síndrome de Abstinência Neonatal no primeiro trimestre, com maior risco no terceiro; vasoconstrição da placenta, reduzindo o fluxo de sangue e nutrientes, resultando em retardo do desenvolvimento fetal e aumento do risco de prematuridade; risco significativo de cardiopatias congênitas, principalmente no primeiro trimestre; e aborto espontâneo (Carvalho, et al., 2020).

A Paroxetina é a droga que foi mais associada a anomalias congênitas se comparadas com as demais drogas da mesma classe, sendo, também, associada às cardiopatias congênitas. É estimado que a dosagem acima de $25 \mathrm{mg} /$ dia dobre o risco para más formações fetais. Quando utilizada no primeiro trimestre, aumenta significativamente os riscos para anencefalia, defeito do septo atrial e obstrução da via de saída do ventrículo direito (Balkowiec-Iskra et al., 2017). A sertralina e citalopram são difundidos como as drogas de maior segurança por apresentarem menos evidências de efeitos adversos, sendo a sertralina mais indicada por diretrizes (Carvalho, et al., 2020; Camacho, et al., 2016) 
Nomura e Pinto e Silva (2007) estimam que 30\% dos RN expostos a terapia com ISRS apresentam síndromes perinatais, sendo caracterizadas por choro, taquipnéia, insuficiência respiratória leve, distúrbios gastrintestinais e cianose. São comparadas, ainda, com os sintomas de algumas síndromes em adultos. Nesse estudo ainda é levantado estatísticas quanto à maior incidência de baixo peso e síndrome da angústia respiratória.

Em questão do não tratamento, Chilsom e Payne (2016) associam ao aumento do risco de prematuridade e baixo peso e ao aumento do risco de suicídio e infanticídio. Essas ideias são confirmadas por Fonseca e Canavarro (2017) que relacionam a TDM não tratada a Depressão Pós-Parto (DPP), pois uma vez que não foi dada importância para esse acometimento psiquiátrico durante o pré-natal, a mulher apresentará os sintomas após o fim da gestação, acrescentam, ainda, a significativa perda de atenção, do sono e da capacidade laboral. Somado a isso, essa mulher poderá apresentar pensamentos negativos e intrusivos quanto ao recém-nascido, podendo transmitir-se em sentimentos de culpa e estranheza, afastamento do papel de cuidadora e até violências contra o mesmo, o que condiz com o aumento do infanticídio.

\section{Conclusão}

Pode-se concluir que existem estudos sobre os benefícios e malefícios do uso de antidepressivos, principalmente os da classe de ISRS seguidos da classe de ISRSN, durante a gestação, de tal forma que dificulta definir se é melhor o tratamento, a adoção de cotratamentos ou o não tratamento, pois à medida que se combate um problema, existe o risco do desenvolvimento de outro. Isso ocorre, pois todas as pesquisas possuem muitos "porém" na apuração dos resultados, há de se considerar muitos vetores e alguns não são possíveis, descaracterizando alguns achados.

Devido a isso, têm-se uma lacuna que deixa margem às interpretações distintas, cabendo aos profissionais definir custo-benefício de acordo com cada paciente e situação. Baseados nos achados, os autores acreditam que estudos clínicos sobre os antidepressivos, principalmente as classes com ação monoaminérgica, deveriam ocorrer com uma amplitude maior e uma rigorosidade metódica tendo em vista determinar um padrão no grau de risco para cada achado dos estudos atuais. Isso, pois, a grande maioria dos artigos relata que um dos grandes problemas das pesquisas são as limitações relacionadas a dosagens, biótipo de cada gestante, subnotificação e subrelevância quando relacionado a outras doenças (principalmente crônicas como diabetes e hipertensão) e alguns outros problemas.

Considerando todos esses pontos, podemos, ainda, sugerir mais pesquisas quanto ao uso dos antidepressivos tricíclicos que demonstraram efeitos adversos de menor impacto na saúde tanto da mulher quanto do RN e de menor incidência (achados esses expostos em alguns dos estudos, porém não muito abordados devido à falta de ensaios clínicos suficientes para serem considerados parâmetros). Somado a tal, a elaboração de protocolos voltados para o atendimento de gestantes com transtornos mentais poderia atrair mais estudos para o uso tanto da terapia farmacológica como da não farmacológica, produzindo uma gama de ações e cuidados pautados no saber científico e com respaldo comprovado para os profissionais que atendem esse público.

\section{Referências}

World Health Organization (WHO) (2020) Depression. https://www.who.int/en/news-room/fact-sheets/detail/depression

Albert, P. R. (2015). Why Is Depression More Prevalent in Women?. Journal of Psychiatry \& Neuroscience, 40 (4), $219-221$.

Gordon, J. L., \& Girdler, S. S. (2014). Hormone replacement therapy in the treatment of perimenopausal depression. Curr Psychiatry Rep., 16 (12), 517.

American Psychiatric Association (2014). Manual diagnóstico e estatístico de transtornos mentais V(DSM-V). Porto Alegre: Artmed

Carvalho, L. A. G. et al. (2020). Tratamento farmacológico da depressão em gestantes: uma revisão da literatura. Brazilian Journal of Health Review, Curitiba, 3(4), 10891-10900. 
Klieman, A., Boing, E., \& Crepaldi, M. A. (2017). Fatores de risco para ansiedade e depressão na gestação: revisão sistemática de artigos empíricos. Mudanças- Psicologia da Saúde, 25(2), 69-76

Silva, B. A. B. et al. (2020). Depressão em gestantes atendidas na atenção primária à saúde. Cogitare enferm. [Internet]; 25. https://dx.doi.org/10.5380/ce.v25i0.69308.

Krob, A. D. et al. (2017). Depressão na gestação e no pós-parto e a responsividade materna nesse contexto. Rev. Psicol. Saúde, Campo Grande, 9(3), 3-16. https://dx.doi.org/10.20435/pssa.v9i3.565

Fonseca, A. D., \& Canavarro, M. C. (2017). Depressão Pós-Parto. In: PROPSICO: Programa de atualização em Psicologia Clínica e da Saúde Ciclo 1. 111164. Porto Alegre: Artmed Panamericana Editora.

Zhao, X., Liu, Q., Cao, S., Pang, J., Zhang, H., Feng, T. et al. (2017). A meta-analysis of Selective Serotonin Reuptake Inhibitors (SSRIs) use during prenatal depression and risk of low birth weight and small for gestational age. J. Affective Disorders, 230, 1-124. https://dx.doi.org/10.1016/j.jad.2018.08.061

Hutchison, S. M., Masse, L. C., Pawluski, J. L., \& Oberlaner, T. F. (2018). Perinatal Selective Serotonin Reuptake Inhibitor (SSRI) Effects on Body Weight at Birth and Beyond: a review of animal and human studies. J. Reproductive Toxicology. https://dx.doi.org/10.1016/j.reprotox.2018.02.004

Guan, H-B., Wei, Y., Wang, L-L., Qiao, C., \& Liu, C-X. (2018). Prenatal Selective Serotonin Reuptake Inhibitor Use and Associated Risk for Gestacional Hypertension and Preeclampsia: a meta-analysis of cohort studies. J. Women's Health. https://dx.doi.org/<10.1089/jwh.2017.6642

Gao, S-Y., Wu, Q-J., Sun, C., Zhang, T-N., Shen, Z-Q., Liu, C-X. et al. (2018). Selective serotonin reuptake inhibitor use during early pregnancy and congenital malformations: a systematic review and meta-analysis of cohort studies of more than 9 milion births. BMC Medicine, 16(205). https://dx.doi.org/10.1186/s12916-018-1193-5

Singal, D., Chateau, D., Struck, S., Lee, J. B., Dahl, M., Derksen, S. et al. (2020). In Utero Antidepressants and Neurodevelopmental Outcomes in Kindergarteners. PEDIATRICS, 145(5).

Masarwa, R., Bar-Oz, B., Gorelik, E., Reif, S., Perlman, A., \& Matok, I. (2019). Prenatal exposure to selective serotonin reuptake inhibitors and serotonin norepinephrine reuptake inhibitors and risk for persistent pulmonary hypertension of the newborn: a systematic review, meta-analysis, and network metaanalysis. American J. Obst. Gyneco. https://dx.doi.org/10.1016/ajog.2018.08.030.

Vallee, J., Wong, Y., Mannino, E., Nordeng, H., \& Lupattelli, A. (2021). Association between Antidepressant Treatment during Pregnancy and Postpartum Self-Harm Ideation in Women with Psychiatric Disorders: a cross-sectional, multinational study. Int J. Environ. Res. Public. Health, 18(46), 2-15. https://dx.doi.org/10.3390/ijerph18010046.

Berard, A., Sheehy, O., Zhao, J-P., Chambers, C., Roth, M., Bozzo, P. et al. (2019). Impact of antidepressant use, discontinuation, and dosage modification on maternal depression during pregnancy. J. Euro. Neuro., 29, 803-812. https://dx.doi.org/10.1016/j.euroneuro.2019.06.007.

Brown, H. K. (2018). Prenatal Selective Serotonin Reuptake Inhibitor Exposure and Child Neurodevelopment: the importance of maternal mental illness. J. American Acad. Child\&Adol. Psyc., 57(3), 150-153. http://dx.doi.org/10.1016/j.jaac.2018.01.002.

Pawluski, J. L., \& Gemmel, M. (2018). Perinatal SSRI medications and offspring hippocampal plasticity: interactions with maternal stress and sex. HORMONES, 17, 15-24. DOI: <10.1007>

Molenaar, N. M., Kamperman, A. M., Boyce, P., \& Bergink, V. (2018). Guidelines on treatment of perinatal depression with antidepressants: na internacional review. Aust.\&New Zeland J. Psych., 52(4), 320-327. http://dx.doi.org/10.1177/0004867418762057.

Field, T., Diego, M., \& Hernandes-Reif, M.(2006). Prenatal depression effects on the fetus and newborn: a review. Infant Behavior \& Development, 29, 445455 .

Chisolm, M. S., \& Payne, J. L. (2016). Management of psychotropic drugs during pregnancy. Thebmj. https://doi.org/10.1136/bmj.h5918.

Field, T., Diego, M., \& Hernandez-Reif, M. (2010). Prenatal Depression Effects and Interventions: a review. Infant Behavior \& Development, 33(4), 409-418. http://dx.doi.org/ 10.1016/j.infbeh.2010.04.005.

Balkowiec-Iskra, E., Mirowska-Guzel, D. M., \& Wielgos, M. (2017). Effect of antidepressants use in pregnancy on foetus development and adverse effects in newborns. Ginekologia Polska, 88(1), 36-42. http://dx.doi.org/10.5603/GP.a2017.0007.

Pearlstein, T. (2013). Use of Psychotropic Medication during Pregnancy and the Postpartum Period. Women'S Health, 9(6), 605-615.

Goracci, A. et al. (2015). Antidepressant use in pregnancy: a critical review of the risk and benefits. Rev. Psichiatr., 50(3), 118-126.

Camacho, R. S. et al. (2006). Transtornos psiquiátricos na gestação e no puerpério: classificação, diagnóstico e tratamento. Rev. Psiquiatr. Clín., São Paulo, 33(2), 92-102.

Nomura, M. L., \& Pinto e Silva, J. L. C. (2007). Riscos e benefícios dos inibidores seletivos da recaptação de serotonina para a depressão durante a gravidez e lactação. Rev. Bras. Ginecol. Obstet., Rio de Janeiro, 29(7). https://doi.org/10.1590/S0100-72032007000700001 .

Mendes, K. D. S., Silveira, R. C. C. P., \& Galvão, M. C. (2008). Revisão integrativa: método de pesquisa para a incorporação de evidências na saúde e na enfermagem. Texto Contexto Enferm., 17(4), 758-764. 